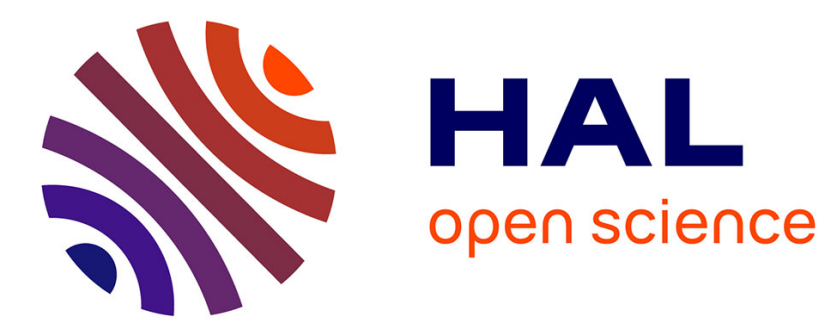

\title{
Les différents visages du confinement des migrants en France \\ Olivier Clochard
}

\section{To cite this version:}

Olivier Clochard. Les différents visages du confinement des migrants en France. Migrations et mutations de la société française, La Découverte, 2014, L'état des savoir, 978-2-7071-7711-7. halshs01098023

\section{HAL Id: halshs-01098023 https://shs.hal.science/halshs-01098023}

Submitted on 23 Mar 2016

HAL is a multi-disciplinary open access archive for the deposit and dissemination of scientific research documents, whether they are published or not. The documents may come from teaching and research institutions in France or abroad, or from public or private research centers.
L'archive ouverte pluridisciplinaire HAL, est destinée au dépôt et à la diffusion de documents scientifiques de niveau recherche, publiés ou non, émanant des établissements d'enseignement et de recherche français ou étrangers, des laboratoires publics ou privés. 


\section{Les différents visages du confinement des migrants en France}

Depuis 1980, période à laquelle le gouvernement de l'époque légalise la rétention administrative et judiciaire des étrangers démunis de documents en règles, de nouveaux modes de confinement des migrants ont été mis en place. La suppression graduelle des contrôles au sein de certaines frontières internes de l'Europe, consécutive à l'application des accords de Schengen (1995) et à l'élargissement de l'Union européenne, a indéniablement renforcé ce processus. Par ailleurs le franchissement des frontières nationales par des étrangers dépourvus des documents exigés et la situation des « sans-papiers » sont de plus en plus associés à des faits délictueux. En conséquence les lieux d'enfermement dont la capacité totale n'a cessé d'augmenter ${ }^{1}$, sont devenus des enjeux importants dans l'évolution de la politique migratoire, les gouvernements successifs ayant procédé à des amendements législatifs pour faciliter la détention des migrants, engendrant dans le même temps d'autres formes de privation de liberté. À Calais et dans les autres villes portuaires de la Manche et la Mer du Nord, les étrangers sont confrontés de façon permanente à des mesures de surveillance, d'exclusion et d'interdiction, et à bien des égards, ces espaces peuvent être considérés comme des antichambres de l'enfermement. Enfin les «nouvelles » technologies comme la numérisation des données relatives à l'identité des migrants et à leur situation administrative occupent une place croissante dans les politiques européennes d'asile et d'immigration, ce sont des mécanismes qui visent à faciliter l'arrestation, l'enfermement et le renvoi d'enfants, de femmes et d'hommes à la recherche de meilleures conditions d'existence.

De par la convergence de leurs fonctionnements et leur substance sociale, ces dispositifs mis en place par les autorités semblent constituer les diverses strates d'un confinement physique et immatériel où d'un côté, les contrôles des migrants doivent constamment être ajustés et transformés voire s'adapter selon les évolutions des flux migratoires «irréguliers », et de l'autre les mobilités et les stratégies des migrants évoluent en fonction de ces nouveaux bornages qui ont été mis en place. Sans entrer dans une démonstration spéculative, il s'agira donc de voir la façon dont ces dispositifs se rattachent à des formes contemporaines d'enfermement visant - selon la terminologie officielle souvent employée - à «mieux gérer les flux migratoires », et de voir comment les usages des centres de rétention administrative, zones d'attente, commissariats, prisons, «jungles»du littoral de la Manche et la Mer du Nord et dispositifs électroniques - marqués par des développements respectifs - se sont diffusés sur le territoire national au cours des vingt dernières années.

\section{Vers une diffusion spatiale des mesures d'enfermement}

Mis en place de façon improvisée aux abords des cinq principaux aéroports durant les années 1980, le nombre de centres de rétention administrative est de neuf en septembre 1992. Aujourd'hui la cartographie de la détention des étrangers est marquée par une constellation de lieux, et ces dispositifs sont «au cœur des politiques migratoires au point de devenir un mode structurel de gestion de la migration » (Migreurop, 2012, p. 79). Mais cet ensemble est marqué par un fonctionnement différencié car selon le type d'établissements où

\footnotetext{
1 De 2000 à 2012, la capacité «d'accueil» connue des lieux d'enfermement (centres et locaux de rétention administrative, zones d'attente) est passé de 723 à 2280 places.
} 
les étrangers sont détenus, les conditions d'accueil ${ }^{2}$ et les règles divergent. La durée de détention peut aller jusqu'à 20 jours pour les personnes interceptées à la frontière et maintenues en zone d'attente ${ }^{3}$, elle est passée de 12 à 45 jours $^{4}$ - entre 2003 et 2011 - pour les étrangers déjà présents sur le territoire et arrêtés au motif de leur seule situation «irrégulière », et pour celles et ceux qui s'opposent à leur renvoi, ils peuvent être condamnés à un an de prison ferme.

Si le nombre de personnes incarcérées pour des motifs liés à l'infraction des règles relatives au séjour des

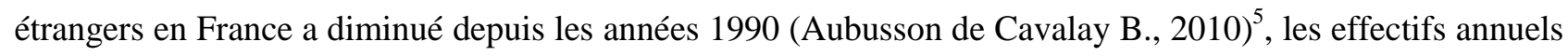
des étrangers maintenus dans des centres de rétention administrative ont augmenté, passant de 17883 en 2000 à 35547 en 2007, avant de diminuer au cours des années suivantes (24 286 en 20116 ). Cette évolution découle de la politique de plusieurs gouvernements des années 2000 qui fixent chaque année des objectifs chiffrés pour les expulsions à réaliser, et elle s'est accompagnée par une augmentation du nombre de centres disposés sur l'ensemble de son territoire. Depuis 2000, quinze établissements ont été construits comme à Nîmes, Rennes ou Metz. Dans certaines villes (Marseille, Toulouse) ces nouveaux centres ont été mis en place pour améliorer les conditions matérielles des personnes détenues dans d'autres structures situées sur d'autres sites. Mais derrière l'amélioration de la situation humanitaire des étrangers enfermés, se dissimulent aussi des considérations économiques (partenariat public privé), fonctionnelles et stratégiques (proximité des aéroports internationaux).

Depuis 1992, les frontières extérieures du territoire sont également concernées avec l'instauration de lieux de détention (zones d'attente), processus qui s'est renforcé de diverses façons au fil du temps. Pour répondre aux « exigences » des politiques migratoires, les autorités ont tout d'abord usé de locaux déjà existants dans les aéroports ${ }^{7}$, gares ferroviaires ou maritimes ouverts au trafic international, ce qu'elles continuent de faire aujourd'hui à certaines frontières en réquisitionnant aussi des chambres d'hôtel le cas échéant ou en maintenant les «passages clandestins » à bord des navires de la marine marchandes en dépit de l'interdiction établie par le Conseil d'Etat en 1998. Le point d'orgue de ce type d'implantation a eu lieu en 2001 avec la construction d'un bâtiment destiné spécifiquement au maintien des étrangers en zone d'attente, situé aux abords des pistes de l'aéroport de Roissy Charles de Gaulle, le centre a une capacité de 174 places. Suite à

\footnotetext{
${ }^{2}$ Le centre de rétention administrative du Mesnil Amelot est le plus grand établissement en France avec 240 places (dont 40 places pour les femmes et les familles), et à l'opposé existe une multitude de locaux de rétention administrative temporaires ou zones d'attente avec une seule place.

${ }^{3}$ À titre exceptionnel, elle peut aller jusqu'à 26 jours lorsque l'étranger dépose une demande d'asile entre le quinzième et vingtième jour de son maintien.

${ }^{4}$ Selon l'article L.552-7 du Ceseda, lorsqu'un étranger a été condamné à une peine d'interdiction du territoire pour des activités liées au terrorisme, « le juge des libertés et de la détention près le tribunal de grande instance de Paris peut, dès lors qu'il existe une perspective raisonnable d'exécution de la mesure d'éloignement et qu'aucune décision d'assignation à résidence ne permettrait un contrôle suffisant de cet étranger, ordonner la prolongation de la rétention pour une durée d'un mois qui peut être renouvelée. La durée maximale de la rétention ne doit pas excéder six mois ».

${ }^{5}$ De 1993 à 2008, le nombre de personnes écrouées pour infraction à la législation de l'entrée et du séjour des étrangers, est passé de plus de 9000 à près de 4000 : des chiffres regroupant aussi bien les étrangers qui se sont opposés à une mesure d'expulsion que les ressortissants des pays de l'UE (Français compris) incarcérés pour infraction à la police des étrangers.

${ }^{6}$ Le nombre s'élève à 51385 si on tient compte des lieux de rétention en outre-mer (Mayotte, Guyane, etc.)

7 À Roissy, deux étages de l'hôtel Ibis ont été utilisés de 1992 à 2000. Ailleurs les autorités ont recours à des pièces situées dans l'enceinte des aéroports comme à Bordeaux et à Nice.
} 
l'arrivée d'exilés sur les côtes méridionales de la France ${ }^{8}$, la loi relative au maintien en zone d'attente a été amendée à deux reprises (2004 et 2011) afin que des lieux pour les nouveaux arrivants puissent être aménagés en tout point du territoire ${ }^{9}$. Cette expansion du dispositif demeure bien souvent théorique mais elle participe à l'idée que le maintien des étrangers peut s'inscrire à tout moment dans les interstices de paysages ruraux ou urbains.

Si les zones d'attente et les centres de rétention administrative sont souvent présentés comme les deux figures de proue de la détention des étrangers en France, les commissariats de police et gendarmerie jouent également un rôle prépondérant dans la détention des étrangers, soit en transformant temporairement ou de façon pérenne, une partie du commissariat en local de rétention administrative, soit en maintenant des étrangers en garde-à-vue avant de les transférer dans des centres de rétention administrative. Pour les locaux de rétention administrative, ce dispositif fut utilisé arbitrairement par les forces de l'ordre jusqu'à la fin des années 1990, puis il a été encadré juridiquement suite à l'adoption d'un décret en 2001. Depuis cette date et jusqu'en 2009, leur nombre a fortement augmenté avant de diminuer suite à plusieurs décisions de justice demandant la libération des détenus pour des motifs relatifs aux conditions inhumaines et dégradantes de leur détention ${ }^{10}$. Concernant la pratique de la garde-à-vue, celle-ci a aussi été remise en cause suite à deux arrêts de la Cour de Justice de l'Union européenne (CJUE) dans les affaires El Dridi et Achughbabian, interdisant la pénalisation de l'irrégularité du séjour ${ }^{11}$. Pour remplacer la garde-à-vue de 48 heures des «sans-papiers » devenue illégale, les élus ont donc légiféré en créant une procédure spécifique de retenue de seize heures maximale afin de vérifier si l'étranger est en règle avec le droit au séjour (article L.611-1-1 du Ceseda) ${ }^{12}$. Ainsi avec une durée plus courte de rétention ou «garde-à-vue » - tout n'est qu'une question de mot - les 5000 commissariats demeurent des éléments clefs, des outils fonctionnels au service des politiques de contrôles des flux migratoires, et notamment dans la région Nord-Pas-de-Calais.

\section{Les jungles de Calais : antichambres de l'enfermement?}

En regardant les chiffres des infractions aux conditions générales d'entrée et de séjour des étrangers en France, on constate qu'entre 2005 et 2010, 22 à $30 \%$ des personnes mises en cause - selon les années -

\footnotetext{
${ }^{8}$ Durant la nuit du 17 février 2001 plus de 900 étrangers, essentiellement kurdes, débarquent du navire East-Sea qui s'est échoué sur les plages du Var, entre Saint-Raphaël et Fréjus. Le 22 janvier 2010, 124 Kurdes originaires de Syrie ont été arrêtés et enfermés par la police après avoir été débarqués près de Bonifacio (Corse).

${ }^{9}$ Ainsi en 2004, suite à l'affaire de l'East-Sea, il a été prévu qu'une zone d'attente puisse être située à proximité d'un lieu de débarquement. Et en 2011, suite à l'arrivée des 124 Kurdes en Corse, le Ceseda prévoit désormais que si « un groupe d'au moins dix étrangers vient d'arriver en France en dehors d'un point de passage frontalier, en un même lieu ou sur un ensemble de lieux distants d'au plus dix kilomètres, la zone d'attente s'étend, pour une durée maximale de vingt-six jours, du ou des lieux de découverte des intéressés jusqu'au point de passage frontalier le plus proche ».

${ }^{10}$ Le ministère de l'Immigration indiquait par ailleurs dans un communiqué en janvier 2010, vouloir ne plus utiliser 17 locaux de rétention administrative, notamment ceux implantés dans les villes de Nancy, Toul, Mont Saint-Martin (Meurthe-et-Moselle), Rennes, Le Havre, Dieppe, Toulon, Laval, Delle (Moselle), Sens, Auxerre, Saint Brieuc, Reims, Charleville-Mézières, Chartres, Montargis et Cercottes (Loiret).

${ }^{11}$ Cour de Justice de l'Union européenne (CJUE) n ${ }^{\circ} \mathrm{C}-61 / 11$ du 28 avril 2011 «El Dridi », CJUE n ${ }^{\circ}$ C-329/11 du 6 décembre 2011 « Achughbabian ».

${ }^{12}$ Code de l'entrée et du séjour des étrangers et du droit d'asile. L'article 78-3 du code de procédure pénale permet également de retenir pendant quatre heure (ou huit heures à Mayotte) une personne française ou étrangère, qui « refuse ou se trouve dans l'impossibilité de justifier de son identité », et ce afin de vérifier son identité.
} 
l'étaient dans la région Nord-Pas-de-Calais ${ }^{13}$. L'importance de ces chiffres - pour une seule des 22 régions métropolitaines - est certes liée à la présence d'exilés qui cherchent à traverser la Manche pour rejoindre la Grande-Bretagne, mais elle souligne surtout l'activité policière qui perdure depuis la destruction du camp de Sangatte en décembre 2002, activité qui a été consolidé avec la signature du traité du Touquet le 4 février 2003 permettant à la Grande Bretagne et à la France de renforcer leurs contrôles migratoires dans le Calaisis. Ainsi en plus des unités de la police aux frontières françaises et anglaises présentes sur les sites d'Eurotunnel et des ports de Calais et Dunkerque, chaque jour dans les rues de Calais, des compagnies de CRS contrôlent, arrêtent ${ }^{14}$ et conduisent des étrangers - en situation « irrégulière » ou parfois avec des papiers en règle - dans les commissariats les plus proches voire dans le centre de rétention administrative (CRA) de Coquelle. Parfois il arrive que les exilés soient transférés dans des CRA situés dans d'autres villes comme à Nîmes, suite à la destruction de jungle comme celle très médiatisée du 22 septembre 2009. Peut-on considérer pour autant la région de Calais - dont la situation migratoire est devenue un des emblèmes de la politique migratoire française - comme un lieu de confinement? Les divers processus d'aliénation auxquels les migrants sont confrontés (difficultés pour manger, se soigner, demander l'asile, avoir un logement dans une structure d'hébergement d'urgence, etc.) conduisent les exilés en transit à adhérer - n'ayant pas d'autres choix - au dispositif des jungles, ces campements informels situés aux abords des aires habitées, dans des bois ou des zones en friche. Et au fil du temps, les jungles semblent être devenus des espaces alternatifs à la détention des migrants dans le sens où ils permettent aux policiers qui tolèrent ces campements informels et leurs recompositions malgré les destructions successives, d'effectuer quasi-quotidiennement des recensements des personnes présentes ${ }^{15}$. Ainsi ces mécanismes de pouvoir et ces formes de gouvernementalité qui s'opèrent depuis plus de dix ans dans les vies des migrants, soulignent bien des formes d'assignation à vivre dans ces campements ${ }^{16}$, soit une situation provisoire de confinement qui précède d'autres plus officielles. Pour exemple les exilés ont certes la possibilité d'aller et venir - comme de nombreux vagabonds ou SDF ressortissants de l'Union européenne - mais leur lieu de vie précaire est bien souvent rattaché à des périodes plus ou moins longues d'enfermement (garde-à-vue, rétention administrative ou prison lorsqu'ils sont considérés comme des passeurs). Le Défenseurs des droits mentionne d'ailleurs «l'allégation de harcèlement constant et quotidien à l'encontre des migrants de la part des forces de l'ordre à travers des contrôles d'identité répétés, des arrestations multiples, des visites, y compris nocturnes, dans des lieux de vie, des destructions et vols de biens, de papiers, des privations d'accès à l'eau, aux soins, à la nourriture, d'insultes raciales et religieuses $»^{17}$. En conséquence ces jungles dont les localisations fluctuent dans l'espace, peuvent être considérés comme les antichambres de l'enfermement administratif des étrangers

\footnotetext{
${ }^{13}$ Bulletin statistique de l'Observatoire national de la délinquance et des réponses pénales, n²6, octobre 2011 . Il n’est pas rare qu'une même personne fasse l'objet de plusieurs arrestations et mise en cellule, parfois dans une seule journée.

${ }^{14}$ Les interpellations s'opèrent dans les campements informels appelés jungles, les maisons ou dépôts squattés par les migrants, sur le lieu de distribution des repas ou sur les chemin menant aux douches ou à la PASS (Permanence d'accès aux soins de santé) voire à proximité des aires d'autoroutes, espaces depuis lesquels les exilés tentent de monter à bord des camions.

${ }^{15}$ Les rapports annuels de la police aux frontières du département du Pas-de-Calais mentionnent ces comptages.

${ }^{16}$ Lors des enquêtes que nous avons effectuées pour le compte de la CFDA (voir bibliographie), plusieurs migrants ont témoigné que des policiers les avaient orienté vers le Calaisis parce qu'il n'avaient pas de titre de séjour en règle.

${ }^{17}$ Décision du Défenseur des droits nº MDS 2011-113, 13 novembre 2012, p.8 [disponible sur Internet]
} 
dans le sens où la majeure partie des arrestations s'opère dans ces lieux. Et avec la destruction quasi-continue des campements informels à Calais et ses alentours, d'autres jungles ont été créées ailleurs sur le littoral de la Manche et la Mer du Nord (Cherbourg, La Havre, Dieppe, Dunkerque), à l'intérieur des terres comme à Norrent-fontes ou Angres voire à Paris.

\section{Maintenir sous surveillance des éléments de la population migrante}

Si les zones d'attente, les centres de rétention administrative et les jungles constituent à la fois la matrice et la pointe relativement visible de la lutte contre l'immigration clandestine, il convient aussi de décrire d'autres types d'exercices du pouvoir comme l'usage de l'assignation à résidence qui combinée aux systèmes biométriques et du bracelet électronique, vise à renforcer la géolocalisation sécuritaire des migrants en vue de la préparation à leur éloignement. Nous ne disposons pas de statistique du ministère de l'Intérieur sur l'usage de l'assignation à résidence qui peut être prise pour une durée de six mois, renouvelable. Seuls des exemples révélés par la presse ou des associations souligne que les autorités ont recours à cette mesure de contrainte. C'est ainsi qu'à l'automne 2012, une famille afghane avec deux enfants de trois mois et trois ans et demi - après avoir été placée dans le centre de rétention du Mesnil Amelot - a été maintenue dans un hôtel à Noisiel (Seine-et-Marne) afin de « répondre » aux critiques du Défenseur des droits, médecins, et associations arborant que cette famille ne devait pas être enfermée. Or après leur placement dans l'hôtel, il a été constaté que le confinement de la famille se poursuivait : interdiction de sortir de leur chambre, même pour les enfants, visites interdites, deux policiers devant la porte et plusieurs autres à l'entrée de l'établissement ${ }^{18}$. Si dans ce cas, la présence policière a été fortement renforcée, l'assignation à résidence est plus souvent couplée à l'obligation faite aux migrants de venir signer au commissariat le plus proche de leur lieu d'habitation, une mesure de surveillance qui dépasse la conception «traditionnelle» des modes de rétention et que le ministre de l'Intérieur Manuel Valls souhaite privilégier ${ }^{19}$. Les autorités souhaitent ainsi «humaniser» les conditions qui précèdent les expulsions, les retenues s'opérant en «milieu ouvert» seraient plus humaines pour les familles voire les femmes ou les personnes âgées. Dans de nombreux cas, la détention en centre de rétention administrative paraît inadaptée et démesurée, et les peines de substitution deviennent incontournables face aux nombreuses critiques. Mais en reprenant les travaux d'Olivier Razac à propos des «centres ouverts » vu comme moyen de substitution au système pénal et de gestion des peines, il semble bien que l'assignation à résidence des étrangers «sans papiers » doit être comprise comme un perfectionnement du dispositif d'enfermement existant (centre de rétention administrative) et non comme un progrès «humain ». Selon le philosophe, «il faut aller au-delà des impressions émotionnelles et des affects pour se rappeler sans cesse que ce qu'il y a de plus dangereux dans la violence, c'est sa rationalité » (Razac O., 2008, p.87). Ainsi dans le prolongement de l'assignation à résidence, les autorités peuvent depuis 2011, avoir aussi recours au bracelet électronique qui pourrait permettre aux autorités - lorsqu'elles n'ont pas pu disposer par exemple d'un laissez-passer consulaire dans les temps requis de la durée maximale de rétention

\footnotetext{
${ }^{18}$ Réseau Education Sans Frontière (2012) Une famille afghane menacée de renvoi vers l'Afghanistan via la Hongrie : c'est le droit d'asile qu'on veut expulser! [disponible sur Internet]

${ }^{19}$ AFP (13 mars 2013) «Contre l'immigration clandestine, Valls donne ses ordres aux préfets », Libération.
} 
(45 jours) - de maintenir la personne sous surveillance et de s'assurer ultérieurement de l'exécution de la mesure d'expulsion. Selon le Ceseda, le bracelet électronique permet en effet « de détecter à distance la présence ou l'absence de l'étranger dans le seul lieu désigné par le juge des libertés et de la détention pour chaque période fixée ». On peut aussi penser que les autorités françaises ont imaginé utiliser ce type de moyen pour les personnes relevant du règlement «Dublin II », de 2005 et 2011, le taux de transferts par rapport au nombre de demandeurs d'asile potentiellement réadmissibles vers un autre pays de l'Union européenne était inférieur à $20 \%$. Ainsi le bracelet électronique et les bases de données biométriques renforcent les logiques de traçabilité de certains individus sans complètement exclure les logiques d'enfermement et d'exclusion (qui prévalent dans les établissements fermés), soit une évolution qui engendre un nombre illimité d'espaces de confinement dont les limites seront bien plus difficiles à saisir. Aujourd'hui l'enjeu des expulsions est en train de s'opérer en partie sur ce terrain, le ministère de l'intérieur souhaitant avec certains organismes rechercher des alternatives à la rétention administrative.

\section{Conclusion}

Les mécanismes de sécurisation des politiques migratoires qui se sont tout d'abord traduits par l'émergence de quelques centres de rétention administrative, puis de zones d'attente dans les (aéro)ports et gares ferroviaires ouvertes au trafic international, se sont étendus de manières successives au cours des années 2000 sur l'ensemble du territoire. Ce sont de nouvelles formes d'exercice du pouvoir dans le sens où de nouveaux lieux d'enfermement de migrants peuvent être aujourd'hui mis en place à tout instant, sans que la loi vienne remettre en question leur légitimité ou du moins cette remise en cause est rendue plus difficile. À une autre échelle, la situation migratoire de Calais et ses environs est caractéristique de ces processus de mise à l'écart qui perdurent sous diverses façons depuis près de vingt ans dans la région. Quelques soient leurs formes (établissements fermés ou lieux de mise à l'écart comme les jungles), ce sont des espaces qui cherchent à se fondre dans le paysage afin qu'ils passent inaperçus et s'intègrent à la vie quotidienne des migrants. Et ce processus se renforce avec de nouvelles procédures administratives et judiciaires comme l'assignation à résidence et l'usage du bracelet électronique, les moyens de communications instantanées consolident en effet l'idée de vouloir réaliser un contrôle continu, ces techniques inédites pourraient bien concourir à une nouvelle physionomie du confinement des migrants.

\section{Bibliographie}

Bruno Aubusson de Cavalay in Contrôleur Général des Lieux de Privation de Liberté (2010) Rapport annuel 2009, Contribution : Lieux de privation de liberté en France : éléments de chiffrage, Paris, Dalloz, pp.233273

Coordination française pour le droit d'asile / CFDA (2008) La loi des «jungles ». La situation des exilés sur le littoral de la Manche et la Mer du Nord, [disponible sur Internet]

Mathilde Darley et Nicolas Fischer (dir.) (2010) Le traitement de l'immigration, entre logique administrative et logique pénale, Champ pénal, Vol. VII [disponible sur Internet]

Didier Fassin (dir.) (2010) Les nouvelles frontières de la société française, Paris, La Découverte, 608 p.

Nicolas Fischer (2005) «Clandestins au secret. Contrôle et circulation de l'information dans les centres de rétention administrative français » in Cultures \&Conflits, vol. 57, pp. 91-118, [disponible sur Internet]

Nicolas Fischer (2009) Le corps comme champ de bataille. Politiques de l'humanitaire dans un centre de 
rétention français in Makaremi C., Kobelinsky C., (dir.), Enfermés dehors. Enquêtes sur le confinement des étrangers, Bellecombe-en-Bauges, Le Croquant, pp. 85-102.

Carolina Kobelinsky et Chowra Makaremi (2008) Confinement des étrangers: entre circulation et enfermement, Cultures \&Conflits, ${ }^{\circ} 71$, [disponible sur Internet]

Nadia Khrouz (2010) «Calais et le nord de la France : zone d'errance, porte de l'Angleterre » in Migreurop, Les frontières assassines de l'Europe, pp. 64-87

Stefan Le Couran (2010) Ce que fait la politique de contrôle de l'immigration. De l'étranger menotté au clandestin in Mathilde Darley et Nicolas Fischer, Le traitement de l'immigration, entre logique administrative et logique pénale, Champ pénal, Vol. VII [disponible sur Internet]

Migreurop (2012) Atlas des migrants en Europe. Géographie critique des politiques migratoires, Paris, Armand Colin, $144 \mathrm{p}$.

Olivier Razac (2008) Avec Foucault, après Foucault : Disséquer la société de contrôle, Paris, L'Harmattan, $176 \mathrm{p}$. 